$\mathcal{G}$ eometry $\&$ Topology Monographs

Volume 2: Proceedings of the Kirbyfest

Pages 321-333

\title{
Fermat limit and congruence of Ohtsuki invariants
}

\author{
XiaO-SONG LiN \\ ZHENGHAN WANG
}

\begin{abstract}
By calculating the Fermat limit of certain $q$-Fermat functions, we get explicit surgery formulae for the second and third Ohtsuki invariants for homology 3-spheres. The surgery formula of the second Ohtsuki invariant $\lambda_{2}$, combined with an argument using the general theory of finite type invariants of homology 3 -spheres, leads to a congruence relation between the first and second Ohtsuki invariants: $\lambda_{1}=2 \lambda_{2} \bmod 24$.
\end{abstract}

AMS Classification 57M99; 57N10, 57M50

Keywords Fermat limit, homology 3-spheres, Ohtsuki invariants

Dedicated to Rob Kirby on the occasion of his 60th birthday

\section{Introduction}

In [11], Ohtsuki extracted a series of rational topological invariants denoted by $\lambda_{1}, \lambda_{2}, \ldots, \lambda_{n}, \ldots$ of oriented homology 3 -spheres from the $S O(3)$ quantum invariants of Reshetikhin and Turaev [15, 3]. Physically, they correspond to the coefficients of the asymptotic expansion of Witten's Chern-Simons path integral at the trivial connection as shown by Rozansky [14]. This construction stimulated a series of works on finite type invariants of homology 3-spheres.

The purpose of this article is to present the following theorem about a congruence relation between the first and second Ohtsuki invariants:

Theorem 1.1 For every oriented homology 3-sphere $M$, we have

$$
\lambda_{1}(M)=2 \lambda_{2}(M) \quad \bmod 24 .
$$

Remark It is known that $\lambda_{1}(M) / 6$ is equal to the Casson invariant of $M$ [10]. Here the Casson invariant $\lambda(M)$ is normalized such that $\lambda=1$ for the Poincaré homology 3 -sphere $\Sigma(2,3,5)$ (+1-surgery on the right trefoil knot). 
It is also known [9] that $\lambda_{2}(M) \in 3 \mathbb{Z}$ for every $M$ (a simpler proof will be given here in Section 5). So the congruence (1) could also be expressed as

$$
\frac{\lambda_{1}(M)}{6}=\frac{\lambda_{2}(M)}{3} \quad \bmod 4 .
$$

The proof of the congruence (1) goes roughly as follows: In [9], we introduced the notion of Fermat functions and Fermat limit. By an explicit calculation of the Fermat limit of a certain $q$-Fermat function involving weighted Gauss sums, we get a surgery formula for $\lambda_{2}$. The congruence (1) is then first proved using the surgery formula in the case when $M$ is obtained from Dehn surgery on a knot in $S^{3}$.

Explicit surgery formulae for $\lambda_{n}$ were also given in [9]. These formulae were used to prove that $\lambda_{n}$ is a finite type invariant of order $3 n$ in [4]. See also $[13]$ and $[5,6]$. We then use the general machinery of finite type invariants of homology 3-spheres to argue that the congruence (1) for homology 3-sphere obtained from Dehn surgery on knots implies that it holds for all homology 3 -spheres.

We originally expressed our congruence in the form (2). When Rob Kirby saw it, he suggested that we put it in the form (1) because of the significance of 24 in the study of 3-manifolds. He also asked whether there are general congruence relations among $\lambda_{1}, \lambda_{2}, \ldots, \lambda_{n}$ similar to (1). We are unable to have any hint about his question even for $\lambda_{3}$ because the formulae are rather involved.

It is possible that the congruence (1) is related with the connection between modular forms and quantum invariants of 3-manifolds suggested in [7].

The article is organized as follows: We will outline some calculation in [9] leading to a surgery formula of $\lambda_{n}$ in Sections 2, 3, 4. The reader will notice that Formula (3) is better expressed here than in [9], and Formula (6) (the surgery formula for $\lambda_{3}$ ) is new here. In Section 5 we will prove Theorem 1.1 using surgery formulae for $\lambda_{1,2}$ and the theory of finite type invariant of homology 3 -spheres.

Acknowledgements The first author is supported in part by an NSF grant and the second author is supported by an NSF postdoctoral fellowship.

\section{Fermat Functions and Their Residues}

We fix some notation that will be in force throughout this article. Let $\mathbb{P}$ denote the set of all odd prime numbers, and $\mathbb{Q}$ the rational numbers. 
Given $r \in \mathbb{P}$,

- $\mathbb{Z}_{r}$ is the ring of $r$-adic integers.

- We use $\bar{m}$ to denote the integer in $\{1, \cdots, r-1\}$ such that $m \cdot \bar{m}=1$ $\bmod r$.

- We will use $q$ to denote the $r$-th root of unity, $e^{2 \pi \sqrt{-1} / r}$. The quantum integer $[k]_{q}$ is

$$
[k]_{q}=\frac{q^{\frac{k}{2}}-q^{-\frac{k}{2}}}{q^{\frac{1}{2}}-q^{-\frac{1}{2}}} .
$$

- For $R=\mathbb{Z}$ or $\mathbb{Z}_{r}, O\left((q-1)^{k} ; R\right)$ stands for a complex number of the form $u(q-1)^{k}$ for some $u \in R[q]$.

- The Gauss sum is $G_{0}(q)=\sum_{k=0}^{r-1} q^{k^{2}}$. The weighted Gauss sum is $G_{2 l}(q)=\sum_{k=0}^{r-1} k^{2 l} q^{k^{2}}$.

Definition 2.1 Suppose $f$ is a function $f: \mathbb{P} \rightarrow \mathbb{Q}$ such that $f(r) \in \mathbb{Z}_{r}$ for all sufficiently large $r, f(r)$ is a Fermat function if there exists a rational number $\lambda=m / n$ (in simplified form) independent of $r$ such that when $f(r)=m^{\prime} / n^{\prime}$ (in simplified form), then $m^{\prime} n=m n^{\prime} \bmod r$ for all sufficiently large $r$.

If $f$ is a Fermat function, we will call the rational number $\lambda$ the residue of $f$ and denote it by $\operatorname{Res}(f)$.

Lemma 2.2 The residue of a Fermat function is unique.

Proof If there is another rational number $\lambda^{\prime}$ such that $f(r)=\lambda^{\prime} \bmod r$ for all sufficiently large primes $r$, then the numerator of the rational number $\lambda-\lambda^{\prime}$ will be divisible by all sufficiently large primes $r$. Therefore, $\lambda-\lambda^{\prime}=0$, ie $\lambda=\lambda^{\prime}$.

A typical Fermat function comes from Fermat's little theorem (and hence the name). For any rational $a \neq 0$, the function $f(r)=a^{r-1}$ is a Fermat function with $\operatorname{Res}(f)=1$. There are many other Fermat functions. For example, the function $f(r)=(r-1) / 2$ is a Fermat function whose residue is $-1 / 2$. Also, the function $f(r)=(r-1)$ ! is a Fermat function because of Wilson's theorem, which says that $(r-1) !=-1 \bmod r$ [2]. On the other hand, an example of a non-Fermat function is given by $f(r)=\left(\frac{r-1}{2}\right)$ !. See [2] for a discussion of the residue of $\left(\frac{r-1}{2}\right)$ !, which turns out to depend on $r$ in a quite complicated way.

Lemma 2.3 The set of all Fermat functions is a ring over the rationals $\mathbb{Q}$. 
This follows easily from the following facts.

Lemma 2.4 Suppose that $f$ and $g$ are Fermat functions. Then

(1) for rational numbers $\alpha$ and $\beta, \alpha f+\beta g$ is a Fermat function whose residue is $\alpha \cdot \operatorname{Res}(f)+\beta \cdot \operatorname{Res}(g)$;

(2) $f \cdot g$ is a Fermat function whose residue is $\operatorname{Res}(f) \cdot \operatorname{Res}(g)$;

(3) if $\operatorname{Res}(g) \neq 0$, then $f / g$ is a Fermat function with residue $\operatorname{Res}(f) / \operatorname{Res}(g)$.

Thus, in particular, every polynomial function of $r$ with rational coefficients is a Fermat function whose residue is its constant term. And every rational function of $r$ with rational coefficients is a Fermat function if the constant term of the denominator is not zero. Its residue is the value of the function at $r=0$. More generally, we have

Lemma 2.5 Suppose $F(r)=\frac{P(r)}{Q(r)}$ is a rational polynomial of $r$, and $f(r)$ is a Fermat function with residue $\lambda$. If $Q(\lambda) \neq 0$, then the composite function $F(f(r))$ is a Fermat function.

We give another example of Fermat functions and the details can be found in $[11,9]$.

Example 2.6 For a fixed integer $k$, the function

$$
D_{k}(r)=\frac{\left(\frac{r-1}{2}\right) !}{\left(\frac{r-1}{2}-k\right) !}
$$

is a Fermat function whose residue is given by

$$
\operatorname{Res}\left(D_{k}\right)= \begin{cases}\left(-\frac{1}{2}\right)\left(-\frac{1}{2}-1\right) \cdots\left(-\frac{1}{2}-(k-1)\right) & \text { for } k>0 \\ 1 & \text { for } k=0 \\ \frac{1}{\left(-\frac{1}{2}+1\right) \cdots\left(-\frac{1}{2}-k\right)} & \text { for } k<0 .\end{cases}
$$

Recall that $f(r)=\left(\frac{r-1}{2}\right)$ ! is not a Fermat function.

\section{$3 q$-Fermat Functions and Fermat Limit}

The $S O(3)$ quantum invariants of 3 -manifolds take values in $\mathbb{Z}[q]$ when appropriately normalized. We like to study the so-called perturbative expansion 
of these invariants. This leads us to think of $q$ as a variable, and study the expansion around $q=1$. In this section, we describe the $q$-analogue of Fermat functions when the function takes values in $\mathbb{Z}_{r}$ adjoint with the $r$-th root of unity $q, \mathbb{Z}_{r}[q]$. It is reasonable to expect that the corresponding object of residue lies in the ring $\mathbb{Q}[[t-1]]$ of formal power series in $t-1$ with coefficients in $\mathbb{Q}$, where $t$ is a formal variable in place of $q$.

Let $c(r)$ be an integer sequence indexed by $r$ with

$$
\lim _{r \rightarrow+\infty} c(r)=+\infty \quad \text { and } \quad c(r) \leq r-2 .
$$

Given a complex function $f_{q}(r)$ on $\mathbb{P}$ which takes values in $\mathbb{Z}_{r}[q]$. Fix a nonnegative integer $n$. For sufficiently large $r$, we write

$$
\begin{aligned}
f_{q}(r)= & a_{r, 0}+a_{r, 1}(q-1)+\cdots+a_{r, n}(q-1)^{n}+ \\
& \cdots+a_{r, c(r)}(q-1)^{c(r)}+O\left((q-1)^{c(r)+1}, \mathbb{Z}_{r}\right)
\end{aligned}
$$

for some $a_{r, n} \in \mathbb{Z}_{r}$.

Notice that although the expansion $f_{q}(r)$ is not unique as $q$ is not a free variable, $a_{r, n} \in \mathbb{Z} / r \mathbb{Z}$ is well defined for $0 \leq n \leq c(r)$. This is because of $r=O((q-$ $\left.1)^{r-1} ; \mathbb{Z}\right)$, and the relation in $\mathbb{Z}_{r}[q]: \sum_{i=0}^{r-1} q^{i}=0$. Thus, if $a_{r, n}$ is a Fermat function, its residue depends only on the function $f_{q}(r)$.

Definition 3.1 The complex function $f_{q}(r)$ has a Fermat limit, denoted by f-lim $f_{q}(r)$, if each $a_{r, n}$, thought as a function of $r$, is a Fermat function with $\operatorname{Res}\left(a_{r, n}\right)=\lambda_{n}$. By definition,

$$
\text { f-lim } f_{q}(r)=\sum_{n=0}^{\infty} \lambda_{n}(t-1)^{n} \in \mathbb{Q}[[t-1]] .
$$

We will call $f_{q}(r)$ a $q$-Fermat function if its Fermat limit exists.

The Fermat limit of $f_{q}(r)$ is well-defined and if it exists, it is unique. The uniqueness of a Fermat limit follows from the uniqueness of residues of Fermat functions (Lemma 2.1).

The following are some basic properties of the Fermat limit.

- The $q$-analogues of both Lemma 2.2 and Lemma 2.3 hold.

- If $f(r)$ is a Fermat function which takes values in $\mathbb{Z}$ with residue $\lambda$, then $q^{f(r)}$ is a $q$-Fermat function whose Fermat limit is $t^{\lambda}$, where it is understood that $t^{\lambda}$ is expanded as a power series of $t-1$. 
Let

$$
\tilde{G}_{2 l}(q)=(q-1)^{l} \frac{G_{2 l}(q)}{G_{0}(q)} .
$$

The following theorem (see [9] Lemmas 6.1 and 6.2) is very important for our study of the $S O(3)$ quantum invariants of 3-manifolds.

Theorem 3.2 $\tilde{G}_{2 l}(q)$ is a $q$-Fermat function and

$$
\mathrm{f}-\lim \tilde{G}_{2 l}(q)=A_{l} \cdot\left(\frac{t-1}{\log t}\right)^{l}
$$

where $A_{l}=\left(-\frac{1}{2}\right)^{l}(2 l-1) ! !=\left(-\frac{1}{2}\right)^{l} \cdot 1 \cdot 3 \cdots(2 l-1)$, and $A_{0}=1$.

One may compare (3) with

$$
\frac{\int_{-\infty}^{\infty} x^{2 l} e^{-x^{2}} d x}{\int_{-\infty}^{\infty} e^{-x^{2}} d x}=(-1)^{l} A_{l} .
$$

\section{Surgery Formulae of Ohtsuki invariants}

Let $L=K_{1} \cup K_{2} \cup \cdots \cup K_{\# L}$ be an oriented link in $S^{3}$, where we use \#L to denote the number of components of $L$. We will denote the unknot by $O$, and the empty link by $\emptyset$.

The Conway polynomial $\nabla(L ; z) \in \mathbb{Z}[z]$ is defined by

$$
\left\{\begin{array}{l}
\nabla(O ; z)=1 \\
\nabla(\emptyset ; z)=0 \\
\nabla\left(L_{+} ; z\right)-\nabla\left(L_{-} ; z\right)=-z \cdot \nabla\left(L_{0} ; z\right)
\end{array}\right.
$$

Here, as usual, $L_{+}, L_{-}$and $L_{0}$ are the links which have plane projections identical to each other except in one small disk where their projections are a positive crossing, a negative crossing and an orientation preserving smoothing of that crossing, respectively. Note the negative sign on the right hand side of the skein relation. Its effect is to change the usual Conway polynomial by a normalization factor $(-1)^{\# L-1}$. So for a knot $K, \nabla(K ; z)$ is the same as the usual Conway polynomial of $K$. In particular

$$
\nabla(K ; z)=1+c_{2}(K) z^{2}+c_{4}(K) z^{4}+\cdots+c_{2 k}(K) z^{2 k} .
$$


The Jones polynomial $V(L ; t) \in \mathbb{Z}\left[t^{\frac{1}{2}}, t^{-\frac{1}{2}}\right]$ is defined by

$$
\left\{\begin{array}{l}
V(O ; t)=1 \\
V(\emptyset ; t)=\left(t^{\frac{1}{2}}+t^{-\frac{1}{2}}\right)^{-1} \\
t V\left(L_{+} ; t\right)-t^{-1} V\left(L_{-} ; t\right)=\left(t^{\frac{1}{2}}-t^{-\frac{1}{2}}\right) V\left(L_{0} ; t\right) .
\end{array}\right.
$$

Note that our normalization differs from the usual definition of the Jones polynomial. Actually it is obtained from the usual Jones polynomial by changing $t$ to $t^{-1}$ and multiplying by $(-1)^{\# L-1}$. We put

$$
X(L ; t)=\frac{V(L ; t)}{\left(t^{\frac{1}{2}}+t^{\frac{-1}{2}}\right) \# L-1} .
$$

Then

$$
X(O ; t)=X(\emptyset: t)=1
$$

We also put

$$
\Phi(L ; t)=\sum_{L^{\prime} \subset L}(-1)^{\# L-\# L^{\prime}} X\left(L^{\prime} ; t\right)
$$

where the sum runs over all sublinks of $L$ including the empty link and $L$ itself. We have $\Phi(O ; t)=0$ and define $\Phi(\emptyset ; t)=0$.

Further, we put

$$
\Phi_{i}(L)=\left.\frac{d^{i} \Phi(L ; t)}{d t^{i}}\right|_{t=1}
$$

so that

$$
\Phi(L ; t)=\sum_{i=0}^{\infty} \frac{\Phi_{i}(L)}{i !}(t-1)^{i} .
$$

Finally for each $i \geq 1$, we set

$$
\phi_{i}(L)=\frac{(-2)^{\# L}}{(\# L+i) !} \cdot \Phi_{\# L+i}(L) .
$$

The invariants $\phi_{i}$ are the basic link invariants we use to express $\lambda_{n}$.

A link is called an algebraically split link (ASL) if the linking number between every pair of components is 0 . In particular, a knot is always an ASL. A framed ASL is said to be unit framed if the framing of each component is \pm 1 . Given a link $L$ and a positive integer $m$, we use $L^{m}$ to denote the 0 -framed $m$ parallel of $L$, ie, each component in $L$ is replaced by $m$ parallel copies having linking number zero with each other. So $L^{m}$ is an ASL if $L$ is. When $L$ is ordered, sublinks of $L^{m}$ will be in one-one correspondence with $\mu$-tuples $\left(i_{1}, \ldots, i_{\mu}\right)$, where $\mu=\# L$, in such a way that $L^{\prime}$ has $i_{\xi}$ parallel copies of 
the $\xi$-th component of $L, 0 \leq i_{\xi} \leq m$. If $L$ is a framed link, $L^{m}$ and all its sublinks will inherit a framing from $L$. If the $\xi$-th component of $L$ is framed by $f_{\xi}, \xi=1, \ldots, \mu$, we denote

$$
f_{L}=\prod_{\xi=1}^{\mu} f_{\xi}
$$

The existence of Ohtsuki invariants is based on two key ingredients. The first one is the following property of Jones polynomial for cablings:

Lemma 4.1 (Ohtsuki, Proposition 3.4 in [11]) As a power series in $t-1$,

$$
\Phi\left(L^{\mathbf{i}} ; t\right)=O\left((t-1)^{|\mathbf{i}|+\max (\mathbf{i})}\right) \in \mathbf{Q}[[t-1]]
$$

if $L$ is an ASL. Here if $\mathbf{i}=\left(i_{1}, \cdots, i_{\sharp L}\right)$, then $|\mathbf{i}|=i_{1}+\cdots+i_{\sharp L}$, and $\max (\mathbf{i})$ is the largest of $i_{j}$.

This implies that the following formal power series is well-defined for every ASL:

$$
\tilde{\Phi}(L)=\sum_{l=0}^{\infty} \sum_{L^{\prime} \subset L^{l} \backslash L^{l-1}} \frac{\Phi(L ; t)}{(t-1)^{\sharp L^{\prime}}}
$$

Another key ingredient is the existence of the Fermat limit of the following function:

$$
\begin{aligned}
& H_{i, f}(q)=(-f) \cdot\left(\frac{f}{r}\right) \cdot q^{3 \cdot \overline{4} \cdot f-\overline{2}} \cdot \frac{(q-1)^{i+1}}{G_{0}(q)} \\
& \left\{\sum_{k=1}^{\frac{r-1}{2}} q^{\overline{4} \cdot f\left(k^{2}-1\right)}[k]_{q} \sum_{j=0}^{\frac{k-1}{2}}(-1)^{j}\left(\begin{array}{c}
k-j-1 \\
j
\end{array}\right)\left(\begin{array}{c}
k-2 j-1 \\
i
\end{array}\right)[2]_{q}^{k-2 j-1}\right\},
\end{aligned}
$$

where $G_{0}(q)$ is the Gauss sum and $\left(\frac{f}{r}\right)$ is the Legendre symbol. Note that $H_{i, f}(q) \in \mathbb{Z}_{r}[q]$.

The existence of the Fermat limit of $H_{i, f}(q)$ is established by Ohtsuki [11], and our computation of this limit is the key to the explicit formulae of Ohtsuki invariants. Our computation relies on Theorem 3.2.

Suppose $L$ is a unit-framed ASL, then the $S O(3)$ quantum invariant of the oriented homology 3 -sphere $S_{L}^{3}$ can be expressed as follows:

$$
\tau_{r}\left(S_{L}^{3}\right)=\sum_{l=0}^{\frac{r-3}{2}} \sum_{L^{\prime} \subset L^{l} \backslash L^{l-1}} \frac{\Phi\left(L^{\prime} ; q\right)}{(q-1)^{\sharp L^{\prime}}} \cdot H_{\mathbf{i}\left(\mathbf{L}^{\prime}\right), \mathbf{f}}(q),
$$


here $\mathbf{i}\left(\mathbf{L}^{\prime}\right)$ is the corresponding $\sharp L$-tuple of $L^{\prime}$. For $\mu$-tuples $\mathbf{i}=\left(i_{1}, i_{2}, \cdots, i_{\mu}\right)$, and $\mathbf{f}=\left(f_{1}, f_{2}, \cdots, f_{\mu}\right), H_{\mathbf{i}, \mathbf{f}}=\prod_{j=1}^{\mu} H_{i_{j}, f_{j}}$.

Now let $\mathrm{f}$-lim $H_{i, f}(q)=H_{i, f}(t)$. Then as a power series of $t-1$, we have

$$
\begin{aligned}
\mathrm{f}-\lim \tau_{r}\left(S_{L}^{3}\right) & =\sum_{l=0}^{\infty} \sum_{L^{\prime} \subset L^{l} \backslash L^{l-1}} \frac{\Phi\left(L^{\prime} ; t\right)}{(t-1)^{\sharp L^{\prime}}} \cdot H_{\mathbf{i}\left(\mathbf{L}^{\prime}\right), \mathbf{f}}(t) \\
& =1+\lambda_{1}(t-1)+\lambda_{2}(t-1)^{2}+\cdots
\end{aligned}
$$

Explicit surgery formulae for $\lambda_{i}, i=1,2,3$ have been worked out using the above formulation. We need the following calculation:

- $H_{0, f}(t)=1$

- $H_{1, f}(t)=(-f) \cdot(t+1)$

- $H_{2, f}(t)=f \cdot \frac{(t+1)^{2}}{2} \cdot\left[1+f+4 \sum_{m=1}^{\infty} g_{1, m}(t-1)^{m-1}\right]$

- $H_{3, f}(t)=(-f) \cdot \frac{(t+1)^{3}}{6} \cdot t^{\frac{1+f}{2}} \cdot\left[1+24 \cdot \sum_{m=0}^{\infty} g_{1, m+3}(t-1)^{m+1}\right]$,

where $g_{i, j}$ are constants determined by

$$
\sum_{m=0}^{\infty} g_{l, m}(t-1)^{m}=A_{l} \cdot\left(\frac{t-1}{\log t}\right)^{l}, \quad l \geq 0
$$

(both sides are power series of $t-1$ ).

Theorem 4.2 Let $L$ be a unit framed ASL and $S_{L}^{3}$ be the homology 3-sphere obtained from Dehn surgery on $L$. Let $\lambda_{i}$ be the $i$-th Ohtsuki invariant. Then

$$
\lambda_{1}\left(S_{L}^{3}\right)=\sum_{L^{\prime} \subset L} f_{L^{\prime}} \phi_{1}\left(L^{\prime}\right)
$$

and

$$
\lambda_{2}\left(S_{L}^{3}\right)=\sum_{L^{\prime} \subset L} f_{L^{\prime}} \phi_{1}\left(L^{\prime}\right) \frac{\# L^{\prime}}{2}+\sum_{L^{\prime} \subset L^{2}} f_{L^{\prime}} \phi_{2}\left(L^{\prime}\right) \frac{1}{2^{s_{2}\left(L^{\prime}\right)}}
$$

and

$$
\begin{gathered}
\lambda_{3}\left(S_{L}^{3}\right)=\sum_{L^{\prime} \subset L} f_{L^{\prime}} \phi_{1}\left(L^{\prime}\right) \frac{\# L^{\prime}\left(\# L^{\prime}-1\right)}{8} \\
+\sum_{L^{\prime} \subset L^{2}} f_{L^{\prime}} \phi_{2}\left(L^{\prime}\right) \frac{s_{1}+2 s_{2}+\frac{1}{3} \sum_{i_{\xi}=2} f_{\xi}}{2^{s_{2}+1}}+\sum_{L^{\prime} \subset L^{3}} f_{L^{\prime}} \phi_{3}\left(L^{\prime}\right) \frac{1}{2^{s_{2}+s_{3}} \cdot 3^{s_{3}}} .
\end{gathered}
$$

Here, if $L^{\prime}$ corresponds to the $\mu$-tuple $\left(i_{1}, \ldots, i_{\mu}\right), s_{j}\left(L^{\prime}\right)=\#\left\{i_{\xi} ; i_{\xi}=j\right\}$. 
See Theorem 5.1 in [9]. The formula (6) is new here. It is proved following the same but more tedious calculation used in the proof of (4) and (5). So we will not repeat it here.

In the rest of this section, we describe the invariants $\lambda_{1}, \lambda_{2}$ for homology $3-$ spheres obtained from $1 / n$-Dehn surgery on knots.

Let $c_{4}$ be the coefficient of $z^{4}$ in the Conway polynomial of $K$ and $v_{i}$ be the $i$-th derivative of $V\left(K ; e^{h}\right)$ at $h=0$.

Theorem 4.3 Let $n$ be an integer, and $S_{K, 1 / n}^{3}$ be the homology 3-sphere obtained from $1 / n-D e h n$ surgery on a knot $K$. Then

$$
\lambda_{1}\left(S_{K, 1 / n}^{3}\right)=-n \cdot v_{2}(K)
$$

and

$$
\lambda_{2}\left(S_{K, 1 / n}^{3}\right)=\frac{n}{2} v_{2}(K)-\frac{n}{3} v_{3}(K)+n^{2}\left[v_{2}(K)+\frac{5}{3} v_{2}^{2}(K)-60 c_{4}(K)\right] .
$$

Some examples are as follows.

Example 4.4 (i) If $M$ is the Poincaré homology 3-sphere $\Sigma(2,3,5)(+1-$ surgery on the right-handed trefoil knot), then $\lambda_{1}(M)=6$ and $\lambda_{2}(M)=39$.

(ii) If $M$ is the homology 3 -sphere $\Sigma(2,3,7)(+1$-surgery on the left-handed trefoil knot), then $\lambda_{1}(M)=6$ and $\lambda_{2}(M)=63$.

We may check that the congruence (1) or (2) holds in these examples.

\section{Proof of Theorem 1.1}

We first consider the special case when $M=S_{K, 1 / n}^{3}$.

Lemma 5.1 For every knot $K, v_{2}(K) \in 6 \mathbb{Z}$ and $v_{3}(K) \in 36 \mathbb{Z}$.

Proof Using the fact that the knot invariants $v_{2}$ and $v_{3}$ are of finite type (see eg [1]), we only need to check the desired congruences on the (left-handed) trefoil knot (for $v_{2}$ ) as well as on the figure- 8 knot (for $v_{3}$ ). We have

- $V\left(\right.$ terfoil $\left.; e^{h}\right)=e^{h}-e^{4 h}+e^{3 h}$;

- $V\left(\right.$ figure- $\left.8 ; e^{h}\right)=1+e^{-2 h}+e^{2 h}-e^{-h}-e^{h}$. 
Thus, $v_{2}$ (trefoil) $=1-4^{2}+3^{2}=-6, v_{3}$ (trefoil) $=1-4^{3}+4^{3}=-36$, and $v_{3}($ figure- 8$)=(-2)^{3}+2^{3}-1^{3}-(-1)^{3}=0$.

By Lemma 5.1 and Equations $(7,8)$, we see $\lambda_{1}(M)=2 \lambda_{2}(M) \bmod 24$ if $M=S_{K, 1 / n}^{3}$. We also see $\lambda_{2} \in 3 \mathbb{Z}$ here.

If $M=M_{1} \# M_{2}$, we have

$$
\begin{aligned}
& \lambda_{1}(M)=\lambda_{1}\left(M_{1}\right)+\lambda_{1}\left(M_{2}\right), \\
& \lambda_{2}(M)=\lambda_{2}\left(M_{1}\right)+\lambda_{2}\left(M_{2}\right)+\lambda_{1}\left(M_{1}\right) \lambda_{1}\left(M_{2}\right) .
\end{aligned}
$$

So, the desired congruences hold for connected sums of homology 3-spheres obtained from surgery on knots.

We consider the general case now.

There is a parallel theory of finite type invariants for oriented homology 3spheres (see $[12,13,8])$ and Ohtsuki invariants $\lambda_{n}$ are known to be finite type invariants of order $3 n$. In particular, for any ASL with $\sharp L>6$, we have

$$
\sum_{L^{\prime} \subset L}(-1)^{\sharp L^{\prime}} \lambda_{1,2}\left(S_{L^{\prime}}^{3}\right)=0 .
$$

So essentially, we only need to calculate $\lambda_{1,2}$ and check the congruences on the homology 3 -sphere $M_{*}$ obtained from +1 surgery on a 6-component link $L$ coming from the graph $\phi$ in the following way: First replace each edge with a circle, and then hook the circles together like Borromean rings for each tri-valent vertex.

A direct calculation of $\lambda_{2}$ on $M_{*}$ involves the computation of the Jones polynomial of a 12-component link with 96 crossings, a task too tedious to pursue.

Instead, let us first blow down four circles in $L$ to get a two component link $J \cup K$. Both components $J$ and $K$ are now knotted so that we cannot blow down further. But the link $J \cup K$ has the following nice property:

There are collections of circles $\left\{C_{1}, C_{2}, C_{3}, C_{4}\right\}$ (each $C_{i}$ contains two circles) in the complement of $J \cup K$ so that

(1) $C_{1} \cup C_{2} \cup C_{3} \cup C_{4}$ is a trivial link;

(2) the link $J \cup K \cup C_{1} \cup C_{2} \cup C_{3} \cup C_{4}$ is an ASL and is surgery equivalent to a separate link $\left(J \cup C_{1} \cup C_{2}\right) \amalg\left(K \cup C_{3} \cup C_{4}\right)$;

(3) blowing down all circles in any non-empty subset of $\left\{C_{1}, C_{2}, C_{3}, C_{4}\right\}$ will unknot either $J$ or $K$. 
Therefore, the values of $\lambda_{1,2}$ on $M_{*}$ are equal to a linear combination (with integer coefficients) of that of $\lambda_{1,2}$ on several homology 3-spheres obtained from surgery on knots, and a connected sum of such homology 3-spheres, respectively. Thus, $\lambda_{2} \in 3 \mathbb{Z}$ and $\lambda_{1}=2 \lambda_{2} \bmod 24$ also hold. This finishes the proof of Theorem 1.1 as well as the fact that $\lambda_{2} \in 3 \mathbb{Z}$ for all homology 3 -spheres.

The existence of such collections of circles $\left\{C_{1}, C_{2}, C_{3}, C_{4}\right\}$ will be quite obvious once we have a good picture of $J \cup K$. It is a good exercise in the so-called Kirby calculus, which is a wonderful tool Rob Kirby invented for our benefit. And we will leave the exercise to those who has not yet had enough chance to play with this tool.

\section{References}

[1] D Bar-Natan, On the Vassiliev knot invariants, Topology, 34 (1995) 423-471

[2] G Hardy, E Wright, An introduction to the theory of numbers, Oxford University press, Fifth Edition (1979)

[3] R Kirby, P Melvin, The 3-manifold invariants of Witten and ReshetikhinTuraev for sl(2,C), Invent. Math. 105 (1991) 473-545

[4] A Kricker, B Spence, Ohtsuki's invariants are of finite type, J. Knot Theory Rami. (1997) 583-597

[5] R Lawrence, Asymptotic expansion of Witten-Reshetikhin-Turaev invariants for some simple 3-manifolds, J. Math. Phys. 36 (1995) 6106-6129

[6] R Lawrence, On Ohtsuki's invariants of 3-manifolds, preprint (1997)

[7] R Lawrence, D Zagier, Modular forms and quantum invariants of 3manifolds, Asian J. Math. 3 (1999) 93-108

[8] X-S Lin, Finite type invariants of integral homology 3-spheres: a survey, from: "Knot Theory" (V Jones, J Kania-Bartoszynska, J Przytycki, P Traczyk, and V Turaev editors), Banach Center Publications, 42 (1998) 205-220

[9] X-S Lin, Z Wang, On Ohtsuki's invariants of integral homology 3-sphere, Acta Math. Sinica, 15 (1999) 293-316

[10] H Murakami, Quantum SU(2)-invariants dominate Casson's SU(2)-invariant, Math. Proc. Camb. Phil. Soc. 115 (1993) 253-281

[11] T Ohtsuki, A polynomial invariant of integral homology 3-spheres, Math. Proc. Camb. Phil. Soc. 117 (1995) 83-112

[12] T Ohtsuki, Finite type invariants of integral homology 3-spheres, J. Knot Theory Rami. 5 (1996) 101-115

[13] T Ohtsuki, Combinatorial quantum method in 3-dimensional topology, Math. Soc. Japan Memoirs, vol. 3 (1999) 
Fermat limit and congruence of Ohtsuki invariants

[14] L Rozansky, Witten's invariants of rational homology spheres at prime values of $K$ and trivial connection contribution, Comm. Math. Phys. 180 (1996) 297324

[15] N Y Reshetikhin, V G Turaev, Invariants of 3-manifolds via link polynomials and quantum groups, Invent. Math. 103 (1991) 547-597

Department of Mathematics, University of California

Riverside, CA 92521, USA

Department of Mathematics, Indiana University

Bloomington, IN 47405, USA

Email: xl@math.ucr.edu, zhewang@indiana.edu

Received: 20 January 1999 Revised: 10 October 1999 